\title{
An Automated Segmentation Method of Kidney Using Statistical Information
}

\author{
Baigalmaa Tsagaan ${ }^{1}$, Akinobu Shimizuํ, \\ Hidefumi Kobatake ${ }^{1}$, and Kunihisa Miyakawa ${ }^{2}$ \\ ${ }^{1}$ Graduate School of Bio-Applications and Systems Engineering, \\ Tokyo University of Agriculture and Technology, \\ 2-24-16 Naka-cho, Koganei-shi, Tokyo 184-8588, Japan \\ \{tsagaan, simiz, kobatake\}@cc.tuat.ac.jp \\ ${ }^{2}$ Department of Radiology, National Cancer Center Hospital, \\ 5-1-1 Tsukiji, Chuo-ku, Tokyo 104-0045, Japan \\ kmiyagaw@ncc.go.jp
}

\begin{abstract}
This paper presents a deformable model based approach for automated segmentation of kidneys from tree dimensional (3D) abdominal CT images. Since the quality of an input image is very poor and noisy due to the large slice thickness, we use a deformable model represented by NURBS surface, which uses not only the gray level appearance of the target but also statistical information of the shape. A shape feature vector is defined to evaluate geometric character of the surface and its statistical information is incorporated into the deformable model through an energy formulation for deformation. Principal curvature on the model surface, which is invariant to rotation and translation, is adopted as a component of the vector. Furthermore, automated positioning procedure of an initial model is presented in this paper. We applied the proposed method to the 33 abdominal CT images whose slice thickness is $10 \mathrm{~mm}$ and evaluated the effectiveness of the proposing method.
\end{abstract}

\section{Introduction}

The segmentation in medical images remains difficult task due to variation in image quality and requires a laborious work especially in three dimensional (3D) images. The medical images are often corrupted by noise which can cause difficulties when applying the conventional methods, such as thresholding and region growing approaches[1]. To address these problems, deformable models have been offering accurate and robust approach, which ensures smoothly connected extracted regions for noisy edges of an image[2]. In more sophisticated deformable models, the prior information about geometrical shape or the location of organs is used to constrain shape and appearance, as well as the statistical variation of these quantities[2,3]. For example, Cootes et al.[4-6] have proposed the active shape model (ASM), which uses PCA to analyze model's shape variation. ASM represents the object shapes by a set of boundary points. Thus some preprocessing, such as adjustment of the point coordinates for the affine transformation of an input images, is necessary. Recently, hierarchically organized models with affine-invariant statistical information, in conjunction with point correspondences are presented, and the results seem to be excellent [7].

The most common example of parameterized deformable model is Fourier model, which can represent the smooth shapes compactly and whose a prior information can 
be derived from probability distributions of the Fourier coefficients [9]. However, it is not easy to deform the model instinctively, especially in the case of $3 \mathrm{D}$ segmentation [10].

In this paper, we present an automated segmentation procedure of kidneys in abdominal CT images. The image quality in our research is very poor and noisy, due to low spatial resolution between slices (Fig.1). In addition, the surrounding organs such as the liver and spleen whose CT values are similar to those of kidney, are very close to or in contact with a part of kidney. Therefore we adopt a deformable model, which uses not only the gray value of the target but also statistical information of the shapes [11]. Our model is defined by NURBS surface in order to achieve easy manipulation and representation of the smooth shapes [12].

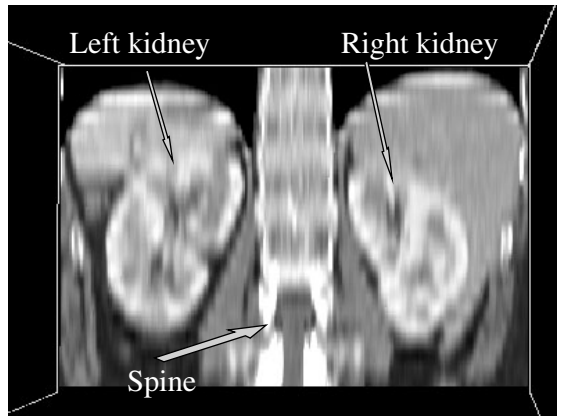

Fig. 1. An example of 3D abdominal CT image (coronal and axial plane)

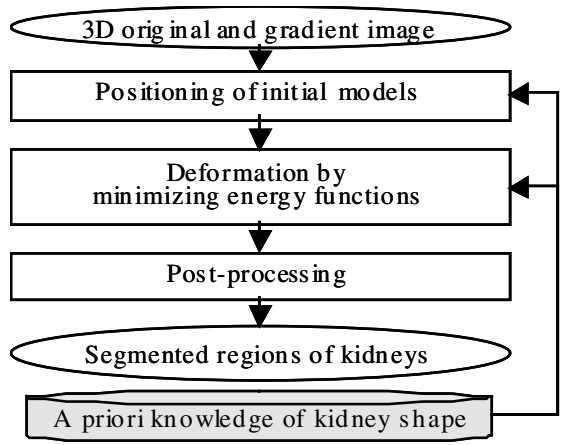

Fig. 2. Flowchart of kidney segmentation process

\section{Method for Segmenting the Kidney}

The flowchart is shown in Fig.2. First, initial models of left and right kidneys are located in an image automatically. Next, we deform the models so that the energy functions can be minimized. Here, statistical information about the shape variation is considered via the energy function. Finally, the post-processing extracts hilum of kidneys.

In the following sub-sections, we first describe the model representation of the kidney. Then the shape feature vector and its statistical information are defined. Next, we present automated positioning procedure of initial models. Finally, definition of the energy function and post-processing are described.

\subsection{Model Description}

A kidney model is defined by a Non-Uniform Rational B-Spline (NURBS) surface,

$$
S(u, v)=\frac{!_{i=0}^{N_{u}-1 N_{v}-1} \prod_{j=0}^{N_{u}-1 N_{v}-1} w_{i, j} B_{i}{ }^{n}(u) B_{j}{ }^{n}(v) P_{i, j}}{\prod_{i=0} \prod_{j=0} w_{i, j} B_{i}{ }^{n}(u) B_{j}{ }^{n}(v)}
$$


where $u, v(\in R)$ are parameters of surface $S(u, v), P_{i, j}$ and $w_{i, j}$ are coordinate vector and weight of control point, respectively. $N_{x}$ is number of control points in the direction $x(=u, v)$, and $B_{x}^{n}$ denotes B-Spline basis function of order $n$ in the direction $x$. The reason of using the NURBS surface is that it has high flexibility for designing a large variety of objects and can be easily modified by moving the control points[12]. The details how we represent kidney's shape by this NURBS surface are given in [13]. An example of kidney models with control points $\left(N_{u}=9, N_{v}=12\right)$ is shown in Fig.3(a). Here, with white boxes are control points, where as black ones are sampling points at regular intervals on the surface model to reduce computational cost of deformation. Shape feature vectors and energy functions are defined with respect to the sampling points, as described in the following subsections.

\subsection{Shape Feature Vector and Its Statistical Information}

Shape feature vector $x$ is defined at each sampling point and its components are principal curvatures of 13 sampling points of interest, as it is shown in Fig.3(b),

$$
x_{U V}=\left(x_{1}, \ldots, x_{26}\right)=\left\{k_{1}\left(S_{U^{\prime} V^{\prime}}\right), k_{2}\left(S_{U^{\prime} V^{\prime}}\right)\right\} \quad\left(U^{\prime}, V^{\prime} \in \eta_{U V}\right)
$$

where $U($ or $V$ ) represents a sampling point number in the direction $u$ (or $v)$. $k_{1}, k_{2}$ are minimum and the maximum of curvatures on the surface point $\left(U^{\prime}, V^{\prime}\right)$, and $\eta_{U V}$ is a set of numbers of neighboring points of the $(U, V)$ th sampling point. Here, it should be noticed that our shape feature vector defined by curvatures remains unchanged under rotation and translation of an input image.

Statistical information about the shape variation is computed as mentioned below. We extracted true surfaces of kidney directly manipulating control points of NURBS model. Shape feature vectors were computed at each sampling point on the extracted surfaces. Then their average vector $\bar{x}_{U V}$ and covariance matrix $\Sigma_{U V}$ of the feature vectors were calculated after deciding the correspondence between control points of different surfaces manually. These statistical information about the geometric feature of the kidney are incorporated into the deformable model via an internal energy function in subsection 2.4 .

\subsection{Positioning of Initial Model}

An initial guess for shape, scale and position of the model can influence the segmentation accuracy significantly. In our early work, we have placed the initial model in an image manually [11]. Here we describe a procedure for estimation of size (scale) of the initial model and the position at which the initial model should be located. Fig.4(a) shows a flowchart of the procedure.

Step1: Extract skin line and spine position by using thresholding.

Step2: Set two ROIs for left and right kidney using spine position and circumscribed rectangular of the skin line, as it is shown in Fig.4(b). The following steps are applied to each of ROIs.

Step3: Estimate the position, where correlation coefficient between CT image and 3D gray template of kidney is maximum. Here, the templates (see Fig.4(c)) are generated 
from the training data set. During the matching process, size of the template is rescaled in order to estimate the scale of the target. To reduce the computational cost, this matching process is applied to the image whose slice interval is $10 \mathrm{~mm}$.

Step4: Estimate more precise initial position by using fine-scale image, which is reconstructed by cubic interpolation (slice interval $=1 \mathrm{~mm}$ ). In this process, we use a mean surface model, which is calculated from the manually segmented regions. First, the mean surface model is rescaled by using the target's scale parameters and place it at the position derived from Step3. Then we move the model to its neighborhood and find the position, where the standard deviation of CT values of all sampling points is minimum.

Step5: Locate the initial models at the searched positions in Step4.

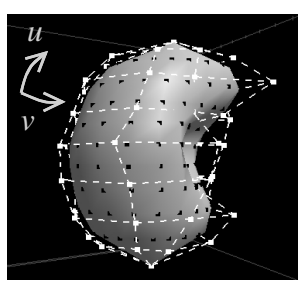

(a)

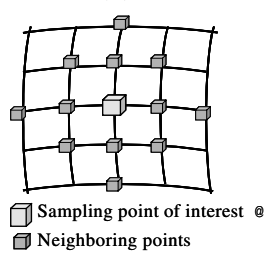

(b)

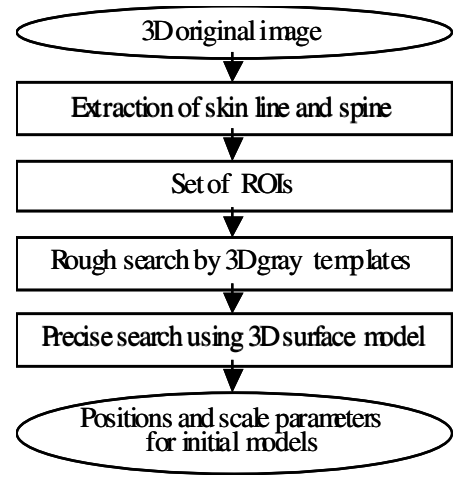

(a)

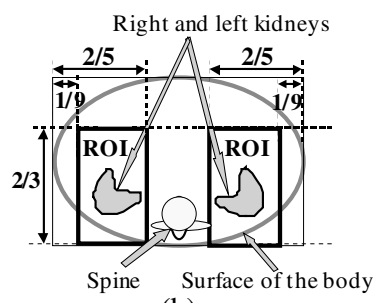

(b)

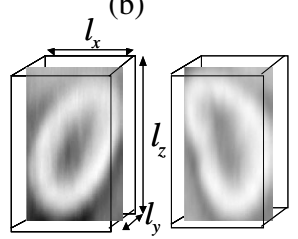

(c)

Fig. 3. (a) An example of kidney model. (b) Neighboring points for shape feature vector

Fig. 4. (a) An automated positioning process for the initial models, (b) Definition of ROIs, (c) Coronal planes of 3D gray templates for left and right kidneys

\subsection{Deformation by Minimizing Energy Function and Post-processing}

The total energy function to be minimized is defined as follows,

$$
E^{i}=E_{\text {ext }}^{i}+w_{\text {int }} E_{\mathrm{int}}^{i} \quad\left(w_{\text {int }} \text { : weight, } i=\text { left, right kidney }\right)
$$

where $E_{\text {ext }}^{i}$ is an external energy and $E_{\text {int }}^{i}$ is an internal energy. A greedy deformation algorithm was used for minimization of energy function [13].

External energy function is defined as follows,

$$
E_{e x t}^{i}=-!_{U=0}^{M_{U}-1} !_{V=0}^{M_{V}-1}\left|\operatorname{Dir}\left(S_{U V}\right) G_{\sigma} * \nabla_{d} f\left(S_{U V}\right)\right|
$$

where $\nabla_{d}$ means a gradient operator with difference distance $d, G_{\sigma}$ is a Gaussian function with standard deviation $\sigma$ and $f$ denotes an original image. Here, function Dir defines the similarity between direction of image gradient vector and that of 
normal vector of the surface. The value ranges from 0 (dissimilar) to 1 (similar). Since the external energy is based on the gradient, it deforms the model to be close to boundary of the object. However, quality of the image is very poor, especially in the direction of body axis. Therefore we prepare two pairs of $\sigma$ and $d$, and deform the model hierarchically to avoid local minima and to reduce computational cost. First, we use large values of $\sigma$ and $d$ to generate a gradient image with broad valley around the boundary and fit the model to the boundary roughly. Then, we use gradient image with small $\sigma$ and $d$, and deform the model to be closer to the boundary.

The sum of Mahalanobis distances of the shape feature vectors is used as $E_{\text {int }}^{i}$,

$$
E_{\mathrm{int}}^{i}=\prod_{U=0}^{M_{U}-1} \prod_{V=0}^{M_{V}-1}\left(x_{U V}-\bar{x}_{U V}\right)^{t} !{ }_{U V}^{-1}\left(x_{U V}-\bar{x}_{U V}\right)
$$

where $M_{U}$ and $M_{V}$ are numbers of sampling points on the surface. Other symbols are explained in subsections 2.1,2.2.

We expect several advantages of using Mahalanobis distance. If the deformed model becomes similar to the mean shape of kidney, internal energy becomes smaller. Our model uses statistical information from the covariance matrix directly, instead of using PCA. Covariance matrix at each point represents the local shape variability around that point. A part of surface with small variation can not be deformed easily, while a part with large variation can be deformed greatly. In addition, our model considers curvature correlation between the neighboring points.

In the post-processing, a region growing method[13], is applied to the region extracted by deformable models and extract the hilum of kidney, where the ureter and vessels connect to kidney in a very complicated way.

\section{Experiments}

The 33 abdominal CT images were used to evaluate the performance of the procedure. The size of image is $512 \times 512 \times 18(\sim 24)$ voxels, the resolution in slice (=size of pixel) is 0.625 (or 0.63 ) $\mathrm{mm}$ and slice thickness are $10 \mathrm{~mm}$. We interpolated gray values to make the isotropic voxel $\left(1 \mathrm{~mm}^{3} /\right.$ voxel). To compute statistical information of left and right kidneys, all images are used.

Segmentation accuracy of extracted region is evaluated by two criterions [11]. First criterion is the degree of correspondence between the segmented region and the true (manually segmented) one, which ranges from 0 and 1 . The high value means that the two regions are overlapped each other. Second criterion is an average distance between the extracted surface and the true one. It ranges from 0 to $\infty$. The small value means that the two surfaces are close to each other.

\subsection{Experimental Results and Discussion}

To evaluate the accuracy of initial positioning process, we measured the distance between a gravity point of an automatically located initial model and that of a manually segmented region. The average displacement was $4 \mathrm{~mm}$ for right kidneys (maximum: $10 \mathrm{~mm}$ ) and $4 \mathrm{~mm}$ for left kidneys (maximum: $9 \mathrm{~mm}$ ). The major direction 
of displacements was the direction parallel to body axis where the image resolution is low. Table1 gives quantitative comparison between extracted regions based on an automatic positioning of initial models and that based on manual positioning. Difference between the evaluation values of two regions are not significant. However it is confirmed that the difference for right kidney is slightly larger than that of left kidney. Main cause of failure is that CT value of the surrounding organs such as liver and spleen are similar to those of kidney.

Fig.5(a),(c) are the segmentation results without the internal energy and Fig.5(b), (d) show the results with the internal energy. Here, left and center figures are axial and coronal slice images, respectively. The black line denotes border of the segmented region, and the white line means border of true one. The deformation with the internal energy avoides local minima and yields a result far better than the deformation without it. Moreover, it is confirmed that the segmentation with the internal energy is robust even in the cases of poor initialization. When we use the internal energy, the average degree of correspondence is approximately $86.5 \%$ and the aforementioned average distance is 1.13 voxels. Both of the values are superior to those of the segmentation without the internal energy and the differences are $2.8 \%$ (maximum: $15.4 \%$ ) and 0.26 voxels (maximum: 1.51 voxel), respectively. In these circumstances, we conclude that the statistical information about the shape works effectively and gives the relatively reliable results. However, the segmentation has failed in some cases where the true contour was affected by the surrounding organs.

Examples of final extracted regions (after post-processing) of left and right kidneys are shown in Fig.6. All results were evaluated by a physician. He observed boundary curves of extracted regions slice by slice and rated them on a 3-point scale: 1=good, 2 =fair, 3=failure. Here "fair" means that the segmentation result is almost good but failure in parts. The 30 of left kidneys were rated to be satisfactorily good or fair, while the remaining three were failure. The 28 of right kidneys were rated good or fair and five were failure. The segmentation accuracy of right kidneys was slightly lower than the left one. The main reason of failure is poor initialization, in conjunction with a notable variation of shape and pose of right kidneys, which is occurred due to stress by liver. The increasing number of training samples will definitely improve the results, in the future.

Table 1. Quantitative evaluation of segmentation results between automatic positioning of initial models and manual positioning. [Average \pm SD.]

\begin{tabular}{lcccc}
\hline \multicolumn{4}{c}{ a. Degree of Correspondence $(\%)$} & b. Average Distance(voxel) \\
\hline Initial Positioning Method & manual & automatic & manual & automatic \\
\hline Left Kidney & $87.3 \pm 2.5$ & $87.1 \pm 2.3$ & $1.08 \pm 0.17$ & $1.09 \pm 0.17$ \\
Right Kidney & $86.5 \pm 2.2$ & $85.7 \pm 3.4$ & $1.14 \pm 0.18$ & $1.18 \pm 0.26$ \\
\hline Both Kidneys & $86.9 \pm 2.3$ & $86.5 \pm 2.8$ & $1.12 \pm 0.18$ & $1.13 \pm 0.22$ \\
\hline
\end{tabular}

\section{Conclusion}

In this paper, we have presented a deformable model for automatically segmenting of kidney. In this model, the shape feature vector is defined at each sampling point on the surface and its statistical information is calculated from a manually segmented 

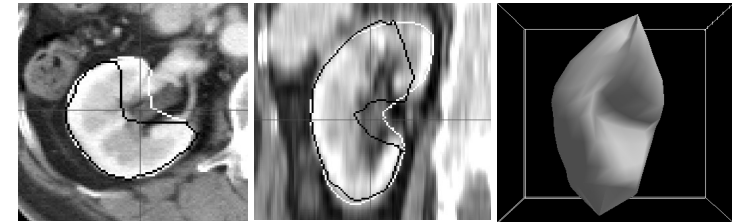

(a) Left kidney of data16 $\left(w_{\text {int }}=0\right)$

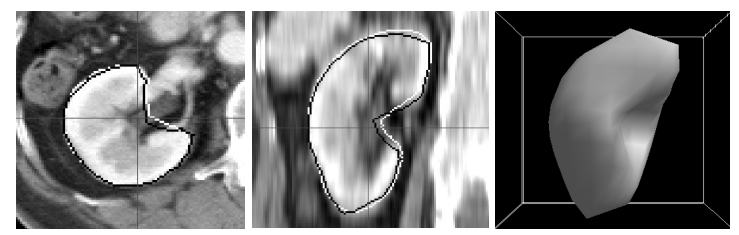

(b) Left kidney of data16 ( $\left.w_{\text {int }}=10\right)$
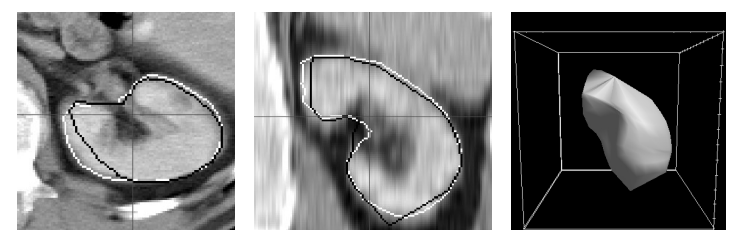

(c) Right kidney of data27 $\left(w_{\text {int }}=0\right)$
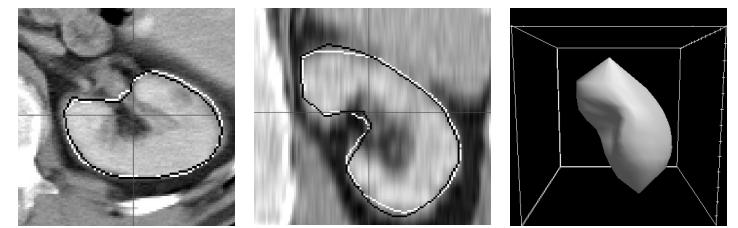

(d) Right kidney of data27 $\left(w_{\text {int }}=10\right)$

Fig. 5. Examples of extracted surfaces. The left and center figures are axial and coronal slice images. Here, the black contour denotes cross curve of segmented region, while the white one denotes the manually segmented one. The right is $3 \mathrm{D}$ resultant surface rendered in perspective

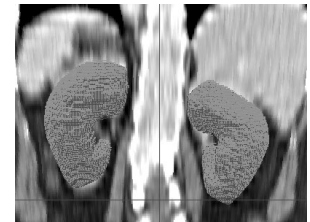

(a) Data16 $\left(w_{\text {int }}=10\right)$

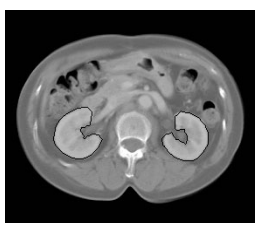

(b) Data16 $\left(w_{\text {int }}=10\right)$

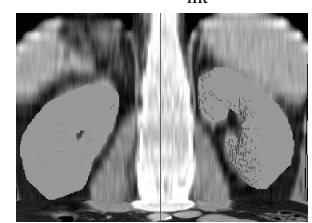

(c) Data27 $\left(w_{\text {int }}=10\right)$

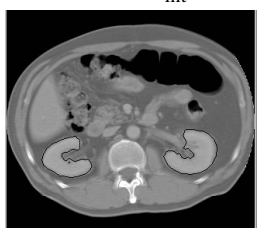

(d) Data27 $\left(w_{\text {int }}=10\right)$

Fig. 6. Examples of extracted regions. (a),(c) Volume region with coronal slice image, (b),(d) Axial slice with extracted curves.

training data. The shape variability is incorporated into the model through formulation of an internal energy. The experimental results in the 3D abdominal CT images were promising. Our current work focuses on extending the proposed model to include statistical information about the global feature of the target, such as pose or orientation [8].

\section{Acknowledgements}

The authors would like to thank colleagues of Kobatake \& Shimizu laboratory for their help and advice. This work is supported by Grant-in-Aid for Scientific Research from Ministry of Education, Culture, Sports, Science and Technology, Japan and Grant-in-Aid for Cancer Research from Ministry of Health, Labour and Welfare in Japan. 


\section{Reference}

1. Duncan,J., Ayache,N.: Medical Image Analysis: Progress Over Two Decades and Challenges Ahead, IEEE Trans. Patt. Anal. Mach. Intell., vol.22 (2000) 85-106

2. McInerney,T., Terzopoulos,D.: Deformable Models in Medical Image Analysis: A Survey, Med. Imag. Anal. vol. 1(2) (1996) 91-108

3. Shimizu,A.: Segmentation of Medical Images Using Deformable Models : A Survey, Med. Imag. Tech. in Japan. vol.20(1) (2002) 3-12

4. Cootes,F., Taylor,L., Cooper,H.: Active Shape Models;Their Training and Application, CVIU vol.61(1) (1995) 38-59

5. Jacob,G., Noble,A., Blake,A.: Evaluating a Robust Contour Tracker on Echocardiographic Sequences, Med. Imag. Anal. vol.3(3) (1998) 63-75

6. Fleute,M., Lavallee, M., Julliard,S.: Incorporating a Statistically Based Shape Model Into a System for Computer-Assisted Anterior Cruciate Ligament Surgery, Med. Imag. Anal. vol.3(3) (1999) 209-222

7. Shen,D., Herskovits,E.H., Davatzikos,C.: An Adaptive- Focus Statistical Shape Model for Segmentation and Shape Modeling of 3-D Brain Structure, IEEE Trans. Med. Imag., vol.20(4) (2001) 257-270

8. Hamarneh,G., McInerney,T., Terzopoulos,D.: Deformable Organisms For Automatic Medical Image Analysis, MICCAI (2001) 66-76

9. Staib,L.H., Duncan,J.S.: Boundary Finding with Parametrically Deformable Models, IEEE Trans. Patt. Anal. Mach. Intell., vol.14(11) (1992) 1061-1075

10. Szekely,G., Kelemen,A. : Segmentation of 2-D and 3-D Objects From MRI Volume Data Using Constrained Elastic Deformations of Flexible Fourier Contour and Surface Models, Med. Imag.Anal., vol.1(1) (1996) 19-34

11. Tsagaan,B., Shimizu,A., Kobatake,H., Miyakawa,K., Hanzawa,Y.: Segmentation of Kidney by Using Deformable Model, ICIP, vol.3 (2001) 1059-1062

12. Terzopoulos,D., Qin,H.,: Dynamic NURBS with Geometric Constraints for Interactive Sculpting”, ACM Trans. Graphics, vol.13(2) (1994) 103-136

13. Tsagaan,B., Shimizu,A., Kobatake,H., Miyakawa,K.: Development of Extraction Method of Kidneys From Abdominal CT Images Using 3-D Deformable Model, Trans. of IEICE in Japan, vol.J85(D-II) (2002) 140-148

14. Williams,D., Shan,D., Shan,M.,: A Fast Algorithm for Active Contours, CVGIP:Imag. Under. , vol.55(1) (1992) 14-26 